\title{
Los niños y la comprensión del mundo mental de los otros: Teorías explicativas
}

\section{Children and their understanding in relation with others' mental world: Explanatory Theories}

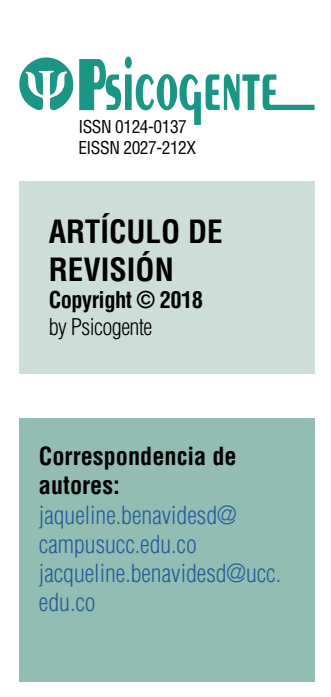

Recibido: $26-01-17$ Aceptado: $17-07-17$

Publicado: $01-07-18$

Jacqueline Benavides Delgado iD
Universidad Cooperativa de Colombia. Santa Marta, Colombia

\section{Resumen}

El objetivo del artículo consistió en revisar, desde el punto de vista teórico, los planteamientos filosóficos y psicológicos que han servido de base para comprender la mente de los Otros por parte de los niños. Se inicia con el concepto de intencionalidad de Brentano (1874-1995), el cual diferencia el mundo físico del mental asumiendo que la mente necesita de objetos para funcionar. Desde la psicología, se abordaron tres teorías: (a) la Teoría de la Simulación o perspectiva de la Primera Persona, propuesta por Gordon (1992), Goldman (1993), Jhonson (1988) y Harris (1995); (b) la Teoría-Teoría o perspectiva de la Tercera Persona, propuesta por Baron-Cohen (2000), Leslie (1987) y Wellman (1995/1990), entre otros, y finalmente, (c) la Teoría de la Intersubjetividad propuesta por Trevarthen $(1974,1979,1998)$ como preámbulo a la propuesta de Gómez $(1998,2005)$ y de Reddy $(2008)$, denominada la perspectiva de la Segunda Persona. Los debates entre estas perspectivas permiten analizar sus diferencias, aportando la posibilidad de enriquecer la mirada acerca de la comprensión de las otras mentes en los niños. En conclusión, se comprende que la teoría de la mente es un concepto que se ha estudiado desde diferentes ópticas, todas las cuales se relacionan a su vez con el concepto de mente.

Palabras clave: mente, teoría de la mente, estados mentales, desarrollo cognitivo, filosofía de la mente.

\section{Abstract}

This paper aims to review the philosophical and psychological approaches as basis for the study related to the ability to understand others' minds by children, based on a theoretical point of views. This paper begins establishing differences between physical and mental world based on the idea that the mind need objects in order to function, according to the concept of intentionality conducted by Brentano (1874/1995). From the psychology point of view three theories are addressed: (a) The Simulation Theory or First Person perspective, introduced by Gordon (1992), Goldman (1993), Jhonson (1988) and Harris (1995); (b) TheoryTheory or Third Person perspective, introduced by Baron-Cohen (2000), Leslie (1987) and Wellman (1995/1990) among others and finally (c) The Intersubjectivity theory introduced by Trevarthen (1974, $1976,1979,1998)$ as a preamble of the proposal introduced by Gómez $(1998,2005)$ and Reddy $(2008)$ called the Second Person perspective. Debates between these perspectives let us to analyze their differences and also, enrich our understanding of others' minds by children. As a conclusion, Theory of Mind has been studied based on different point of views related to the concept of mind.

Key words: mind, theory of mind, mental states, cognitive development, philosophy of mind.

Cómo citar este artículo (APA): Delgado Benavides, J. (2018). Los niños y la comprensión del mundo mental de los otros: Teorías explicativas. Psicogente 21(40), 518-544. https://doi. org/10.17081/psico.21.40.3089 


\section{INTRODUCCIÓN}

El mentalismo insiste en el carácter interno y privado de los procesos mentales, que son inobservables e inaccesibles a los demás. Bajo estos supuestos, entender el mundo mental de Otros e interpretar sus acciones, podría considerarse imposible, pero en lo cotidiano, las personas explican y predicen las conductas de los otros a partir de los estados mentales aparentemente ocultos.

Esta capacidad ha merecido un capítulo importante tanto en la filosofía como en la psicología. Los tratados de filosofía de la mente y las aproximaciones de la psicología del sentido común o psicología popular (en inglés Folk Psychology) han permitido entender que las personas logran comprender su mundo social a partir de los estados mentales propios y de los demás (Martínez-Freire, 1995). La importancia de esta capacidad radica en la posibilidad de integrarse adecuadamente en el mundo social y comprender las intenciones, deseos, conocimientos y creencias de los Otros. Sin esta mirada mental, como sucede en el caso de los niños autistas, el mundo social sería incomprensible (Rivière \& Núñez, 1996).

\subsection{Elementos de una filosofía de la mente}

Tradicionalmente, el concepto de estado mental o fenómeno mental remite al pensamiento filosófico de Brentano (1874/1995). Para este autor, los fenómenos mentales son siempre conscientes y contienen intencionalmente un objeto. De este modo, lo mental siempre se dirige a un objeto: nadie puede querer, sin querer algo, o imaginar sin que el contenido de esta imaginación se dirija hacia algo. La característica de lo mental es, entonces, la intencionalidad. Claramente, la intencionalidad hace referencia al aboutness (en inglés) o en español 'acerca de'. La idea de modelos mentales que representan el mundo surge de esta visión, que concibe el pensamiento como un sistema representacional (Carpendale \& Lewis, 2006). Más en concreto, Brentano (1874/1995) plantea: "Podemos considerar como una definición indudablemente justa de los fenómenos psíquicos la de que son representaciones o descansan en representaciones que les sirven de fundamento" (p.26).

La relación entre las ideas de Brentano y la llamada psicología popular o Folk Psychology radica en que esta mente, accesible a través de la conciencia, que a su vez depende de la intencionalidad, puede pensarse a sí misma y pensar a los otros como seres con mente. En este sentido, como plantea Rivière (2000), el organismo que atribuye mente tiene la capacidad de pensarse a sí 
mismo y atribuir a los otros estados mentales. Según este último autor, esta actividad mental es de orden superior.

\subsection{La teoría-teoría (perspectiva de la tercera persona)}

La Teoría-Teoría (TT) es una postura que nace en la segunda mitad del siglo pasado (Gopnik \& Wellman, 1992) y que ha sido considerada como dominante en este campo. Su principal eje es la metáfora del niño como científico, quien crea hipótesis y conceptos para explicar las acciones de otros a partir de sus estados mentales (Baron-Cohen, 2000; Wellman, 1995/1990). Por lo tanto, para comprender la mente de los Otros, los niños deben construir una teoría (Astington \& Barrault, 2001; Gopnik \& Wellman, 1992; Wellman, 1995/1990). Desde luego, se sabe que existen grandes diferencias entre el sentido común y la ciencia, pero como ocurre en este caso, ¿por qué el conocimiento cotidiano se asimila a una teoría científica? Es claro que el lenguaje, el método y la teorización que se tejen alrededor de un tema para construir una teoría científica, son mucho más elaborados que la explicación ingenua de las acciones humanas, aun así, autores como Wellman (1995/1990) consideran que para comprender la mente de los Otros, los niños construyen una teoría.

En tal sentido, uno de los elementos más característicos de una teoría es la coherencia de los conceptos entre sí. "Por coherente quiero decir que los conceptos y los términos de interés se van entrelazando unos con otros y cada uno proporciona el apoyo necesario para el resto" (Wellman, 1995/1990, p. 24). Un segundo componente descansa sobre distinciones o compromisos ontológicos específicos. En este sentido, existe una interdependencia entre las proposiciones de un mismo campo. Y una tercera condición que implica una teoría es la utilización de principios causal-explicativos.

Como puede verse, Wellman defiende la existencia de una teoría del pensamiento en los niños y asume que ellos, a partir de los 3 años, logran: (a) diferenciar las entidades físicas de las mentales (nivel ontológico), (b) dar explicaciones causales explicativas de las acciones a partir de los estados mentales, y (c) establecer una coherencia entre los conceptos que utilizan para tal fin. En este sentido, el niño puede desarrollar una teoría de la mente (TdM) desde los 3 años, pues solo cuando logra comprender los estados mentales de los Otros o tener una TdM en términos de la TT, cuando logra inferir el estado mental y a partir de este, realiza una predicción de las conductas. 
Otro representante de esta visión es Perner (1994/1991), quien ha desarrollado la idea de la mente como sistema representacional. Justamente de todas estas aproximaciones teóricas se deriva el término $\mathrm{TdM}$, que es al mismo tiempo una de las teorías más robustas y más criticadas.

\subsection{La teoría de la simulación (perspectiva de la primera persona)}

El modelo de Simulación Mental o Perspectiva de la Primera Persona (TS) surge como una alternativa ante la perspectiva de la Tercera Persona. Durante la primera etapa del surgimiento de esta teoría en 1986, su principal interés fue plantear diferentes perspectivas acerca del test de las falsas creencias y de la conceptualización misma de la TT (Brunsteins, 2008). Entre sus planteamientos se destacan dos mecanismos utilizados para comprender las otras mentes: (a) la introspección (Goldman, 1993) y (b) la proyección (Gordon, 1992).

Para Gordon (1992), la simulación permite ponerse en el lugar de la otra persona y proyectarse en su situación. En este sentido, cuando se simula no se imagina al Otro, sino que el yo se proyecta en la experiencia del Otro. Por el contrario, Goldman (1993) considera que para comprender el estado mental del Otro, es necesario fingirlo.

Uno de los principales representantes de la teoría de la simulación fue Johnson (1988), quien consideró que los niños no necesitan construir una teoría para comprender la mente de los Otros. En oposición a ello, este autor defiende que la comprensión de la mente de los Otros se basa en mecanismos intuitivos, con una clara tendencia fenomenológica. Este conocimiento intuitivo está inundado de elementos autorreferenciales, primarios y de primer orden. Según Johnson, la intuición mentalista de los Otros es en realidad una referencia a sí mismo. En este sentido, la capacidad de proyectar e imaginar la propia experiencia en otros es el mecanismo utilizado por el niño para comprender los estados mentales de los Otros. Así, a través de la propia proyección de las experiencias internas es que se entienden las acciones de otros (Gordon, 1986; Harris, 1992; Stich \& Nichols, 1992).

Los teóricos de la simulación ofrecen un ejemplo para comprender su postura: "supongamos que usted quiere predecir cómo un avión se comporta en ciertas condiciones de viento. Una manera de proceder podría ser derivar la predicción de una teoría aeronáutica con la descripción detallada de lo que es un avión. Otra alternativa es construir un modelo del avión y ponerlo en un túnel de viento que reproduce todas las condiciones del viento, y solo 
se observa cómo el modelo se comporta" (Stich \& Nichols, 2003, p.9). Este ejemplo evidencia que para los teóricos de la simulación no es necesario tener un cúmulo de información en la mente para comprender las acciones de los Otros. Lo que se necesita, más bien, es utilizar la propia mente como un modelo de las Otras. De esta manera, como propone Jonhson (1988), "cada uno analiza su propia experiencia, en los zapatos del otro" (p.57).

Harris (1992) amplía esta perspectiva de Johnson (1988) cuando afirma que solo a través de la imaginación se puede comprender la mente de los Otros. Esto no significa que para imaginar los estados mentales de otros, haya que tener experiencias similares, sino que esta se puede sentir a través de la simulación. Por tanto, no es necesario que se compartan los estados mentales tal como se sienten, solo se requiere imaginarlos y simularlos.

En la misma línea anterior, es muy interesante analizar la forma como Harris (1992) rebate las posturas de la TT cuando dice que para el niño no existe esa opacidad ante la mente de los Otros, sino que sus propias experiencias le permiten comprender las experiencias de los demás. La capacidad de ser conscientes de sus estados internos, los lleva a asumir el rol de otros y comprender su mente (Harris, 1992) o, como dice Gordon (1992), a proyectar las propias explicaciones que se le dan a las situaciones. En este sentido, el niño asume el rol del Otro e imagina lo que él pensaría o desearía en la situación del personaje.

Recientemente, los defensores de la TS (Teoría de la Simulación) han admitido que los mecanismos que subyacen a la mindreading son muy diversos y que uno de ellos puede ser la simulación. En concreto, Stich y Nichols (2003) plantean que tanto los mecanismos de información enriquecida (TT) como los de información empobrecida (TS), se utilizan en diferentes situaciones. Por ejemplo, los mecanismos que subyacen a la comprensión de las falsas creencias, las cuales implican atribuir creencias a otros que no son compartidas por la persona, implican mecanismos que se relacionan con el conocimiento de los estados perceptuales de los otros, sea de las verbalizaciones o de información no verbal. En este caso, todos estos aspectos se relacionan con los mecanismos de información enriquecida, más que de simulación. Sin embargo, en otras situaciones, se ve claramente que los mecanismos utilizados son de simulación. Mindreading se define entonces como un fenómeno complejo y multifacético, que puede explicarse por mecanismos de información empobrecida y enriquecida. Este primer intento de integración de la TS y de la TT permite reflexionar acerca de la complejidad de esta capacidad 
$y$, desde luego, de la variabilidad de los mecanismos que se utilizan para comprender la mente de los Otros. Con otras palabras, la anterior reflexión de Stich y Nichols (2003) muestra que la Teoría de la Simulación, o también llamada Perspectiva de la Primera Persona, no puede explicar todas las dimensiones de la comprensión de las otras mentes.

Algunas críticas a la TS se centran, por otra parte, en sus debilidades teóricas. Brunsteins (2008) considera al respecto que la TS carece de aspectos teóricos para comprender la mente de los Otros. En este sentido, su limitante sería que algunos estados mentales podrían comprenderse a través de la simulación, pero no todos.

\subsection{La intersubjetividad y la perspectiva de la segunda persona}

La intersubjetividad fue un término acuñado por Trevarthen (1974, 1976, $1979,1998)$, quien estudió su existencia en bebés y concretamente en los términos de una intersubjetividad primaria y secundaria. Este autor demostró que los bebés interactúan con otros desde los dos meses de edad, en especial con sus madres, mediante un intercambio de miradas, señales, protolenguaje y sonidos, a través de los cuales se establece una conexión afectiva y, desde luego, una comprensión e intercambio de los mutuos estados mentales. En esta línea, los estudios de Gómez $(2004,2005)$ muestran evidencias evolutivas de la capacidad de comprensión de las otras mentes en primates.

Esta perspectiva de la intersubjetividad se ha complementado de forma muy acertada, con los estudios longitudinales realizados por investigadores como Dunn, Brown, Slomkowski, Tesla \& Youngblade (1991). Estos estudiosos han demostrado que existen variables asociadas al desarrollo de la TdM, como son las habilidades lingüísticas. El desarrollo del lenguaje y la exposición a conversaciones con contenido mental predicen, en efecto, posteriores niveles de ejecución en tareas de TdM (Astington \& Jenkins, 1999; Slade \& Ruffman, 2005; Dunn, et. al., 1991). La relación entre el lenguaje y la TdM ha sido estudiada también desde la influencia que los contextos sociales ejercen sobre la comprensión de la mente de los demás. Y autores como Dunn \& Brophy (2005) consideran que la comunicación oral es fundamental para adquirir una TdM, ya que a través de las narrativas y de las historias se comprende la mente de los Otros. Las investigaciones de Dunn han permitido determinar que la participación del niño en conversaciones con contenido mental en los primeros meses de vida (13 meses) predicen el desempeño en tareas de la TdM hacia los 5 años. Por otra parte, además del contenido mental y emocional de las conversaciones, se ha encontrado que la fluidez del 
lenguaje de la madre, así como la participación de los niños en juegos cooperativos con sus hermanos predice un mejor desempeño en la comprensión de las falsas creencias (Dunn, et al., 1991; Hughes \& Dunn, 1998).

Los estudios que han analizado la comprensión de los estados mentales de los Otros y el tipo de relación que existe entre los niños y sus amigos o sus hermanos, han concluido que la relación afectiva que se dé entre ellos determina el tipo de conversaciones mantenidas y sus interacciones. En los estudios longitudinales, por ejemplo, en el realizado por Hughes \& Dunn (1998) probó que los niños que emplean frecuentemente términos mentales en sus conversaciones con los amigos comprenden mejor las falsas creencias $y$, a futuro su ejecución en tareas de la TdM resulta superior. Un ejemplo muy interesante al respecto es la interacción de los niños en los juegos simulados y de imaginación. Los niños solo pueden acceder a este proceso imaginativo compartido, si existe un vínculo afectivo importante entre ellos. Así, las interacciones que los niños tengan con sus iguales en los años del preescolar son predictores de un posterior desempeño en tareas de TdM. Desde luego, estas interacciones sociales tempranas con iguales son espacios privilegiados para que los niños aprendan a asumir el punto de vista de los Otros (Hughes \& Dunn, 1998; Cutting y Dunn, 2006).

Los estudios antes reseñados han sido el preámbulo para el planteamiento de una perspectiva del desarrollo de la TdM centrada en la interacción con los Otros, que se ha denominado Perspectiva de la Segunda Persona (Gómez, 1998, 2004/2007, 2005; Reddy, 2008), surgida en oposición a las posturas de la primera persona (p.e., Harris, 1992) y de la tercera persona (p.e., Wellman, 1990/1995).

Reddy (2008) hace una crítica muy importante a los planteamientos teóricos que hasta ahora se han defendido sobre el origen de la TdM en los niños y plantea la perspectiva de la segunda persona partir de tres supuestos: (a) el rechazo a la idea de la mente opaca, que utiliza los mecanismos de inferencia, imitación o teorización para comprender los comportamientos de los Otros. La inferencia, para Reddy (2008), no puede ser el único mecanismo de acceso a la mente de los otros, puesto que genera incertidumbre sobre la verdad. En consecuencia, en lugar de una mente opaca, Reddy propone la idea de una mente transparente que utiliza mecanismos de percepción. La realidad es que todos los días nos involucramos en interacciones con otros, que implican una lectura de la mente y del cuerpo, y para Reddy (2008), este (el cuerpo) no está separado de la mente; por el contrario, le obedece. En este sentido, para 
Reddy (2008): "La mente es lo que los cuerpos hacen, ellos son públicos, no privados" (p.14). Así, el mismo Reddy concluye que en los niños el comportamiento es la mente, "lo público es un paso necesario para acceder a lo privado y no lo contrario" (p.15). Estas ideas muestran escenarios diferentes y explican que los niños comprenden el mundo social porque el comportamiento hace pública la mente. La idea de una mente que se manifiesta en el cuerpo constituye una de las más importantes diferencias entre la teoría de la segunda persona y las teorías clásicas.

Por otra parte, Reddy (2008) plantea la pluralización de los Otros (b) en contraposición a la mente opaca. La existencia de distintos Otros hace transparente su mente o por lo menos facilita su comprensión. De este modo, la relación previa con el Otro lleva a una comprensión distinta de la mente y, desde luego, esto conlleva la necesidad de estudiar las interacciones diferenciales con cada persona y generalizar la existencia o no de una capacidad en el niño de comprender las otras mentes, solo a partir de una interacción con alguien en especial. En esta línea, Reddy (2008) considera la existencia de una segunda y tercera personas, lo que en sus palabras significa que hay personas con vínculos afectivos (segundas personas) y hay Otros, como cuando se nombra un él o un ella (terceras personas). Así, la experiencia de recibir una sonrisa de alguien considerado en el marco de la segunda persona, es decir, que es significativo a nivel social y afectivo, es una situación muy diferente y genera reacciones y comprensiones sociales muy distintas a aquellas que se presentan cuando el niño recibe de un tercero la misma sonrisa.

Finalmente, Reddy (2008) añade a esta lectura de la mente de los Otros la existencia de vínculos que constituyen un elemento emocional relacionado con la comprensión de las Otras mentes (c). En este sentido, no solo se transmite y se percibe información: la segunda persona implica construcciones basadas en experiencias vinculares.

Según Reddy (2008), las teorías clásicas (Teoría-Teoría y Teoría de la Simulación) difícilmente pueden explicar cómo los niños, antes de los dos años, realizan acciones que denotan la comprensión de los estados mentales de los demás. No cabe duda de que la conexión afectiva entre la madre y su hijo o entre los padres y sus hijos es única y que en esas relaciones se evidencia un diálogo de emociones difícilmente repetible en otros contextos. Es así como en lo cotidiano y en las relaciones cercanas, los niños muestran una mente que dialoga con las otras mentes y las comprende. 
Los cuestionamientos de Reddy (2008) hacia los mecanismos utilizados para entender la mente de los otros ilustran muy bien lo limitado de estas visiones. Por una parte, la visión de la TT que describe una mente opaca y solo accesible desde la inferencia y la conceptualización de estados mentales; por otro lado, la postura de la simulación, que aparece como es, una construcción del Yo y por el Yo. A través de la representación supuesta en esta última, el Otro solo puede ser percibido como parte del Yo. En cambio, la propuesta de la postura de la segunda persona se basa en el reconocimiento del Otro como diferente y no como similar a sí mismo. En este sentido, se da un diálogo entre el Yo y el Otro basado en la interacción, no en la representación que el Yo tiene del Otro, y este diálogo le da existencia al Otro como alguien que interactúa y responde a las acciones del Yo y no solo a la representación que dicho Yo tiene de él. Los mecanismos propuestos en la segunda persona se basan en conceptos previamente planteados por Trevarthen (1998) desde la perspectiva de la intersubjetividad, como se explicó anteriormente. Sin embargo, Reddy (2008) añade un mecanismo basado en la percepción. Así, esta autora considera que la TT plantea un mecanismo basado en la percepción del otro, con una brecha que solo se resuelve a través de la inferencia, y que en la Teoría de la Simulación el mecanismo de la propiocepción es fundamental para comprender las otras mentes. Esto implica la conexión consigo mismo y la percepción de lo que ocurre internamente. En cambio, en la perspectiva de la Segunda Persona, existe una combinación de la propiocepción y la percepción cuando el otro pertenece al plano de la segunda persona o al plano donde el Otro es vincular. La brecha del otro se rompe y se vinculan dos mecanismos: (a) percepción y (b) propiocepción.

Esta visión de la forma como se desarrolla la comprensión de la mente de los otros no se presenta como una percepción (tercera persona) ni como, una propiocepción (primera persona), sino como una sola entidad que involucra tanto la percepción como la propiocepción. Esta postura considera, entonces, que la relación es lo que determina la experiencia que se tiene de los Otros, lo cual implica que solo por la interacción con Otros es posible comprender sus mentes, no mediante un proceso proyectivo, como lo plantea la TS, ni por la inferencia (TT).

En esta misma línea, resulta importante analizar los estudios de Gómez $(1998,2004 / 2007,2005)$ quien, desde sus estudios con primates, reconoce la importancia de la intencionalidad. Gómez plantea dos tipos de intersubjetividad: La intersubjetividad de una vía y la intersubjetividad de dos vías. La primera se refiere a la interacción entre dos, pero uno es objeto de la subjeti- 
vidad del Otro en contraposición con la intersubjetividad de doble vía, donde las dos partes entran en contacto. Definitivamente, la intersubjetividad de una sola vía alude a los mecanismos de la TT y de la Teoría de la Simulación. Por el contrario, la intersubjetividad de doble vía alude a los mecanismos emocionales y atencionales.

\subsection{Debates actuales}

Indiscutiblemente, si se quiere entender cómo los niños comprenden la mente de Otros no se puede dejar de lado la interacción. Pensar en un niño cuya mente trata de entender el mundo social sin que el mundo social tenga algún grado de influencia en esta comprensión, es algo difícil de sostener. Sin embargo, durante más de 38 años (1978-2016), la teoría dominante en este campo ha sido la TT que plantea la metáfora del niño como científico (Wellman 1990/1995) o la visión de Perner (1994/1991) desde la representación mental. Las críticas a esta teoría, que provienen de dos vertientes, han sido las mismas, aunque provengan de diferentes escenarios. Por ejemplo, la Teoría de la Simulación con sus representantes Johnson (1988) y Harris (1992), entre otros, postula que el niño no requiere de un proceso de teorización ni conceptualización de los estados mentales, solo necesita ser consciente de su propia experiencia y trasladarla a la de los Otros. Esta mirada fenomenológica al problema de la comprensión de las otras mentes tampoco alcanza una explicación suficientemente coherente con el problema que se aborda. No se puede negar que, a través de la simulación o de la imaginación, los niños comprenden mucho del mundo social, pero tampoco puede entenderse esta dinámica desde mecanismos netamente subjetivos.

El debate es aún más interesante cuando se considera la posición de Raver \& Leadbeater (1993), quienes plantean la existencia de dos vertientes que intervienen para explicar la capacidad de los niños para comprender, explicar y predecir las acciones de los Otros. La primera, marcada por la influencia de la llamada TdM (p.e., Wellman, 1995/1990), defiende la idea de la inferencia como mecanismo para comprender las acciones de otros a partir de sus estados mentales. La segunda vertiente se sitúa en el campo del desarrollo infantil y estudia la capacidad de los niños para interactuar en su mundo social y comprender las intenciones con los demás (Tomasello, 1999), así como para desarrollar acciones de ayuda, empatía y de humor (Dunn, 1988; Reddy, 2008). Estas dos tendencias parecen irreconciliables y resulta paradójico pensar que solo hasta los 3-4 años los niños logran comprender que las acciones de Otros dependen de sus estados mentales (p.e., Wimmer \& Perner, 1983) cuando la cotidianidad muestra lo contrario. La mirada de Raver y Leadbeater 
(1993) muestra que hay un mundo cotidiano por descubrir, donde los niños demuestran sus capacidades, que no siempre pueden mostrar en el laboratorio. Este es un primer escenario de actualidad sobre el tema.

Una segunda aproximación que intenta dar respuesta a esta disyuntiva es la que plantea Martínez (2011). Este autor intenta reconciliar los planteamientos de la intersubjetividad de Trevarthen (1998) y los planteamientos de la TT. Por una parte, defiende los planteamientos de la intersubjetividad como una condición natural, derivada de un proceso evolutivo demostrado en primates (Gómez, 2005). A partir de esta premisa, organiza niveles de intersubjetividad que se basan en el desarrollo del lenguaje. Así considera que existe una intersubjetividad primaria en el niño antes de la aparición del lenguaje y una intersubjetividad secundaria después que el lenguaje hace su aparición. De otro lado, plantea tres niveles de intersubjetividad a través de los cuales el niño se relaciona con los Otros. Por una parte, considera que los niños interactúan con los Otros cara a cara en la etapa pre-verbal (intersubjetividad primaria). En una segunda etapa (intersubjetividad secundaria) aparece una triangulación con los objetos; y en una tercera etapa (intersubjetividad terciaria) surge la Teoría de la Mente.

Nuevas aproximaciones al entendimiento del desarrollo de la comprensión de las otras mentes se insertan en el debate académico. La cotidianidad adquiere importancia y los estudios sobre producción del sentido del humor en niños desde los 9 meses (Benavides, 2013; Naito, 2016; Reddy, 2001) son nuevos escenarios que rescatan la cotidianidad como contexto fundamental para entender la mente social de los niños. Mika Naito, investigador japonés, considera que la teoría de la mente no es un constructo universal y que tampoco se desarrolla de igual forma en todas las culturas. La mirada de este autor plantea un nuevo reto en el estudio de la TdM sobre el que poco se ha hablado en los debates clásicos: la cultura. En 2003, Naito (2003) y Naito y Koyama (2006) encontraron que los niños japoneses desarrollaban más tardíamente su capacidad para comprender las falsas creencias. Y el dato más interesante de estos estudios radica en los mecanismos utilizados para tal fin. Estos autores encontraron que los niños japoneses utilizan como elementos de análisis el comportamiento externo y las normas sociales, dejando de lado el estado mental interno, sin elementos sociales o relacionales.

Estas nuevas perspectivas permiten analizar el desarrollo de la TdM como un proceso que ha trascendido lo aislado e individual, con un niño que teoriza o simula, para asumirla como una capacidad asociada a la interacción cotidiana, 
con variaciones entre las culturas y, por lo tanto, no puede asumirse como un desarrollo universal.

Nota de autores: Este artículo se deriva de la investigación "La emergencia de la producción de acciones de tomar del pelo y su relación con la teoría de la mente en niños de 18 a 48 meses".

\section{REFERENCIAS}

Astington, J. \& Barrault, T. (2001). Children's theory of mind: How young children come to understand that a people have thoughts and feelings. Infance Young Children, 13(3), 1-12. Disponible en https://journals.Iww.com/iycjournal/ Citation/2001/13030/Children_s_Theory_of_Mind__How_Young_Children_ Come.5.aspx

Astington, J. \& Jenkins, J. (1999). A longitudinal study of the relation between language and theory of mind development. Developmental Psychology, 35(5), 1311-1320. Recuperado de http://psycnet.apa.org/doi/10.1037/0012-1649.35.5.1311

Baron-Cohen, S. (2000). Theory of mind and autism: A fifteen years review. En S. Baron-Cohen, H. Tager-Flusberg, \& D. Cohen, Understanding other minds: Perspective from development cognitive neuroscience (pp.102-105).Oxford: Oxford University Press.

Benavides, J. (2013). La emergencia de las acciones de tomar del pelo en niños entre los 9 y 48 meses y su relación con el desarrollo de la Teoría de la Mente. Tesis para optar el Título de Doctorado. Cali: Universidad del Valle.

Brentano, F. (1995). Psychology from an empirical point of view. En T. Rancurello., D. Teral. \& L. McAllister (Trad.). Londres: Routledge. (Trabajo original publicado en 1874).

Brunsteins, P. (2008). Algunas reflexiones acerca de la simulación mental y la perspectiva de la primera persona. Areté Revista de Filosofía XX (1), 7-38. Disponible en http://revistas.pucp.edu.pe/index.php/arete/article/view/552

Carpendale, J. \& Lewis, C. (2006). How children develop social understanding. Oxford, Inglaterra: Blackwell.

Cutting, A. \& Dunn, J. (2006). Conversations with siblings and friends: links between relationship quality and social understanding. Developmental Psychology 24(1), 73-87. http://doi.org/10.1348/026151005x70337

Dunn, J. \& Brophy, M. (2005). Communication, relationships, and individual differences in children's understanding of mind. In J. Astington, \& J. Baird (Eds.), Why Language Matters for Theory of Mind (pp. 186-219). Oxford: Oxford Press.

Dunn, J. (1988). The beginnings of Social Understanding. United States of America: Harvard University Press.

Dunn, J., Brown, J., Slomkowski, C., Tesla, C. \& Youngblade, L. (1991). Young children's understanding of other people's feelings and belief: Individual difference and their antecedents. Child Development, 62(6), 352-1366. https://doi. org/10.2307/1130811

Goldman, A. I. (1993). The psychology of folk psychology. Behavioral and Brain Sciences, 16(01), 15-28. Disponible en http://users.ecs.soton.ac.uk/harnad/ Papers/Py104/goldman.psyc.html

Gómez, J. C. (1998). Do concepts of intersubjeticity apply to non-human primates? En S. Bråten (Ed.). Intersubjective Communication and Emotion in Early Ontogeny (pp.245-259). Cambridge: Cambridge University Press. 
Gómez, J. C. (2004/2007). El desarrollo de la mente en los simios, los monos y los niños. Madrid: Morata

Gómez, J. C. (2005). Joint Attention and the Notion of Subject: Insights from Apes, Normal Children, and Children with Autism. En N. Eilan., C. Hoerl., T. McCormack. \& J. Roeesler (Ed.) Joint attention: communication and other minds. Issues in Philosophy and Psychology (pp.65-84). Oxford: Oxford University Press.

Gopnik, A. \& Wellman, H. (1992). Why the child's theory of mind really is a theory. Mind and Language, 7(1-2), 145-171. http:/doi/.org/10.1111/j.1468-0017.1992. tb00202.x.

Gordon, R. (1986). Folk psychology as simulation. Mind \& Language, 1, 158-170. https://doi.org/10.1111/j.1468-0017.1986.tb00324.x

Gordon, R. M. (1992). The Simulation Theory: Objections and misconceptions, Mind and Language, 7(1-2), 11-34. https://doi.org/10.1111/j.1468-0017.1992. tb00195.x

Harris, P. (1992). From simulation to folk psychology: The case for development. Mind and Language, 7(1-2), 120-144. http:/doi/.org/10.1111/j.1468-0017.1992. tb00201.x

Harris, P.L. (1995). The child's concept of emotion: An introduction. In J. Russell (Ed.) Everyday conceptions of emotions. (pp.283-287). Dordrecht, Holland: Kluwer.

Hughes, C. \& Dunn, J. (1998). Understanding mind and emotion longitudinal association with mental-state talk young friends. Developmental Psychology, 34(5), 1026-1037. Disponible en https://www.ncbi.nlm.nih.gov/pubmed/9779748

Johnson, C. (1988). Theory of mind and the structure of conscious experience. En J. Astington, P. Harris, \& D. Olson (Eds.), Developing theories of mind (pp.47-63). Oxford, Inglaterra: Cambridge University Press.

Leslie, A. (1987). Pretense and representation: The origin of the theroy of mind. Psychological Review, 94(7), 412-426. Disponible en http://psycnet.apa.org/ buy/1988-13495-001

Martínez, M. (2011). Intersubjetividad y teoría de la mente. Psicología del Desarrollo, 1(11), 9-28. Disponible en http://apadea.org.ar/fotos/2012/11/Mart\%C3\%83\%C2\%ADnez-M.-2011-Intersubjetividad-y-Teor\%C3\%83\%C2\%ADa-de-la-Mente-1. pdf

Martínez-Freire, P. (1995). La nueva filosofía de la mente. Barcelona: Gedisa.

Naito, M. (2003). The relation between theory of mind and episodic memory: Evidence for the development of autonoetic conscience. Journal of Experimental Psychology, 85, 312-336. https://doi.org/10.1016/S0022-0965(03)00075-4

Naito, M. (2016). The socio-cultural construction of theory of mind: A critical evaluation of cognitive science from phenomenological perspective. Japanese Journal of Developmental Psychology, 27(4), 288-298. Disponible en http:// www2.fiit.stuba.sk/ kvasnicka/CognitiveScience/Friedenberg_Cognitive\%20 science.pdf

Naito, M. \& Koyama, K. (2006).The development of false beliefe understanding in japanese children. Delay and deviance? International Journal of Behavior Development, 30(4), 290-304. http://dx.doi.org/10.1177\%2F0165025406063622

Perner, J. (1994). Comprender la mente representacional. En M.A. Galmarini (Trad). Madrid: Paidós (Trabajo original publicado en 1991)

Raver, C. \& Leadbeater, B. (1993). The problem of the other research theory of mind and social development. Human Development, 36, 350-367. https://doi. org/10.1159/000278223

Reddy, V. (2008). How infants know minds. Cambridge: Harvard University Press. 
Reedy, V. (2001). Infants clowns: The interpersonal creation of humor in infancy. Enfance, 52(3), 247-256. https://doi.org/10.3917/enf.533.0247

Rivière, Á. (2000). Teoría de la mente y metarrepresentación. En M. Rodríguez. \& P. Chacón, Pensando la mente: Perspectivas en filosofía y psicología (pp.271-324). Madrid: Biblioteca Nueva Editorial.

Riviére, A. \& Núñez, M. (1996). La mirada mental. Buenos Aires: Aique.

Slade, L. \& Ruffman, T. (2005). How language does (and does not) relate to theory of mind: A longitudinal study of sintaxis, semantics, working memory and false belief. British journal of Developmental Psychology, 23, 117-141. http://dx.doi. org/10.1348/026151004X21332.

Stich, S. \& Nichols, S. (1992). Folk Psychology: Simulation a tacit theory. Mind and Language, 7(1), 35-71. https://doi.org/10.1111/j.1468-0017.1992.tb00196.x

Stich, S. \& Nichols, S. (2003). Folk Psychology. En S. Stich, \& T. Warfields. The blackwell guide of philosophy of mind (pp.235-255). Oxford: Blackwell.

Tomasello, M. (1999). The Cultural Origins of Human Cognition. United States of America: Harvard University Press.

Trevarthen, C. (1974). Conversations with a two-month-old. New Scientist. 2, 230-235.

Trevarthen, C. (1976). Descriptive analyses of infant communicative behaviour. En I. $\mathrm{H}$. Schaf--fer, Studies in mother-infant interaction. Londres: Academic Press.

Trevarthen, C. (1979). Instincts for human understanding and for cultural cooperation: Their development in infancy. En M. von Cranach, K. Foppa, W. Lepenies, \& D. Ploog, Human ethology: Claims and limitsof new discipline. Cambridge: Cambridge Unirvesity Press.

Trevarthen, C. (1998). The concept and foundations of infant intersubjectivity. En Braten, S. (Ed) Intersubjective communication and emotion in early ontogeny ( $\mathrm{pp}$. 15-46). Cambridge: Cambridge University Press.

Wellman, H. (1995). Desarrollo de la teoria del pensamiento en los niños. En C. Boulandier (Trad).Madrid: Desclée De Brouwer. (Trabajo original publicado en 1990).

Wellman, H. \& Liu, D. (2004). Scaling of theory of mind task. Child Development, 75(2), 523-541. https://doi.org/10.1111/j.1467-8624.2004.00691.x

Wellman, H. \& Woolley, J. (1990). From simple desires to ordinary belief: the early development of everyday psychology. Cognition, 35, 245-275. Disponible en https://www.ncbi.nlm.nih.gov/pubmed/2364653

Wellman, H., Cross, D. \& Watson, J. (2001). Meta-analysis of theory of mind development:The truth about false belief. Child Development, 72, 655-684. https://doi. org/10.1111/1467-8624.00304

Wimmer, H. \& Perner, J. (1983). Belief about beliefs: Representation and constrain function of wrong belief in young children`s understanding of deception. Cognition, 13, 103-128. https://doi.org/10.1016/0010-0277(83)90004-5 autor o el licenciante. 